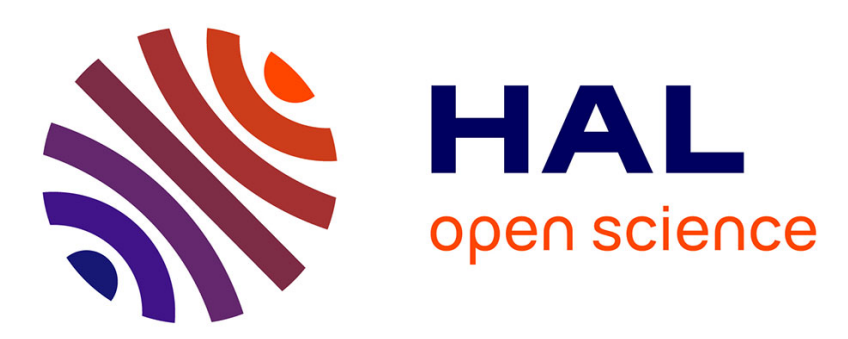

\title{
Increasing vehicles perception using cooperative relaying and priority-based beaconing
} Sanaullah Faiz, Nadjib Achir, Khaled Boussetta

\section{To cite this version:}

Sanaullah Faiz, Nadjib Achir, Khaled Boussetta. Increasing vehicles perception using cooperative relaying and priority-based beaconing. HPSR 2021 - IEEE 22nd International Conference on High Performance Switching and Routing, Jun 2021, Paris, France. pp.1-6, 10.1109/HPSR52026.2021.9481799 . hal-03534746

\section{HAL Id: hal-03534746 \\ https://hal.science/hal-03534746}

Submitted on 21 Jan 2022

HAL is a multi-disciplinary open access archive for the deposit and dissemination of scientific research documents, whether they are published or not. The documents may come from teaching and research institutions in France or abroad, or from public or private research centers.
L'archive ouverte pluridisciplinaire HAL, est destinée au dépôt et à la diffusion de documents scientifiques de niveau recherche, publiés ou non, émanant des établissements d'enseignement et de recherche français ou étrangers, des laboratoires publics ou privés. 


\section{Increasing vehicles perception using cooperative relaying and priority-based beaconing}

\author{
Sanaullah Faiz \\ L2TI, Institut Galillee \\ Universite Sorbonne Paris Nord \\ Villetaneuse, France \\ faiz.sanaullah@edu.univ-paris13.fr
}

\author{
Nadjib Achir \\ L2TI, Institut Galillee \\ Universite Sorbonne Paris Nord \\ Villetaneuse, France \\ nadjib.achir@univ-paris13.fr \\ $T R i B E$ \\ Inria Saclay - Ile de France, France \\ nadjib.achir@inria.fr
}

\author{
Khaled Boussetta \\ L2TI, Institut Galillee \\ Universite Sorbonne Paris Nord \\ Villetaneuse, France \\ khaled.boussetta@univ-paris13.fr
}

\begin{abstract}
The use of vehicle's on-board sensors in increasing the perception of cooperative vehicles is becoming prevailing and essential for the road safety. However trusting only on the on-board sensors could limit the perception of the cooperative vehicles for example an object/vehicle can not be detected if it is impeded by some obstruction. The perception efficiency of vehicles can be increased beyond their field of view (FoV) by exchanging the sensor detected information through V2X communication. The perception of vehicles can be more accurate as much as frequent they share the information. However by transmitting numerous messages could saturate the communication channel and deteriorate the perception improvement specially in the dense circumstances. In this paper we address both these issues and propose two different schemes, (i) an information propagating mechanism to increase the cooperative vehicle's perception above their FoV and (ii) an optimal dynamic cooperative priority based beaconing model to coup with the channel congestion. By using the information propagation mechanism we can increase the perception of cooperative vehicles up to their maximum communication range and on the other end probability based dynamic cooperative priority beaconing scheme selects the most suitable cooperative vehicle to transmit based on its on-board sensor facility. These proposed mechanisms immensely decrease the contention on the channel while extend the perception beyond the FoV. We evaluate both the mechanisms for the performance in terms of vehicles awareness, channel load and communication range through simulations for several vehicle densities. The simulation results show that these proposals provide better performance compare to the classical beaconing schemes.
\end{abstract}

Index Terms-Vehicular safety communications, Cooperative Awareness Messages (CAM), Relaying vehicles perception, priority-based adaptive beaconing.

\section{Introduction}

The research in Vehicle Safety Communications (VSC) is advancing expeditiously towards its actual deployment. In this aspect, the fundamental issue is the system's scalability as it is based on the Dedicated Short Range Communication (DSRC) [2]. In Vehicle Safety Communications, safety is assured through different safety applications which benefit from the pervasive and periodic message broadcasts, also known as Cooperative Awareness Messages (CAM) [14]. All the vehicles broadcast periodic beacons for collective awareness and tracking of the neighbors in the network with the frequency of $10 \mathrm{~Hz}$ recommended in the ETSI-ITS Specifications [3] for vehicle safety communications. This high frequency of beacons create congestion on communication channel even in the medium level networks and emerges data collisions and eventually degrades performance of the overall network. In this context, it is imperative to regulate the beacon's broadcasting frequency without deteriorating the vehicles' awareness.

In this paper, we propose a novel probability-based dynamic cooperative beaconing scheme named Enhanced ECAM (eECAM), benefiting from the work [1] which employ collective perception concept [6] [7] to reduce the transmission frequency of beacons. We consider that the vehicles are equipped with various types of sensor devices like front view cameras, radars, lidars, etc., to collect an explicit view of their environment, thanks to these technological advancements. This precise perception information is then accommodated in the beacon message contents, including the vehicle position and vehicle velocity, and broadcasts in the network. This enhanced cooperative awareness knowledge is then exploited to reduce the transmission of beacons in the network.

Our proposed probability-based dynamic cooperative priority beaconing scheme (eECAM) incorporates an additional cooperative priority strategy for the transmission of beacons along with the Extended CAM (ECAM) mechanism to enhance the performance and reliability. The probability is based on the vehicle's sensor facilities and the sensors' accuracy, which assigns a vehicle a transmission priority at a certain broadcast period. A vehicle owning more and better sensor facilities can have a higher priority to transmit, 
eventually translates into a lower number of transmissions reducing the congestion on the wireless channel. Ultimately this results in an expanded and accurate awareness of the cooperative vehicles in the surrounding yet the Quality of Service (QoS) of the communication network. We have analyzed the performance of eECAM and compared it with the ECAM and standard beaconing scheme of EEE-802.11p (CAM).

The rest of the paper comprises Section-II's literature on the C-ITS and discusses the approaches proposed to cope with the channel congestion in a vehicular environment. The section-III, elaborates the eECAM working mechanism. The methodology and obtained results are discussed in sectionIV. Finally, section-V summarizes the paper contributions.

\section{Related Work}

The [4] proposed an autonomous driving framework where Vehicles share any incident through the base station with a small and large scale. This mechanism broadcasts the information across a large geographical area to enhance network efficiency. In [5], the authors maintain a certain channel load threshold according to the data rate by changing different data rate levels. The data rate step up or step downs a level according to the CBR of the channel.

A multi-state active transmit data rate control mechanism based on DCC has been introduced in [13]. Here the channel load is calculated according to the CBR of the network and a multi-state active DCC protocol chooses the data rate according to the recent channel load.

Authors of [8] has introduced an adaptive transmission power control mechanism and a dynamic packet reception model to cope with the channel congestion using Model Predictive Control (MPC) approach [9] to gain a packet reception rate in the network. The authors have presented a dynamic packet reception model and modeled an adaptive power control algorithm scheme based on the vehicles density and packet reception. In the [11], the authors have used a Distributed fair power adjustment scheme as a congestion control mechanism on the channel. This scheme use the density of the vehicles to apply a dynamic transmission power control strategy for the vehicle safety applications.

The authors of [12], designed a protocol to chooses a minimum transmission power to transmit the CAM for keeping the vehicles connected within a safety zone. The distance between the source vehicle and its nearest neighbors, determine the transmission power. Then a Cooperative piggybacking broaden the awareness beyond the safety zone. In the same way [10] introduces a Random Transmit Power Control method to handle the channel load through data rate and message transmission power. The awareness of the network determines the feasible transmission power and data rate of the channel.

The efforts discussed above based on varying the data rate, transmission power, or contention window size to reduce the contention on the communication channel for the beacons which eventually affects the transmission range and fairness negatively resulting to compromise the awareness of the cooperative vehicles. However, an appropriate mechanism needs to increase the quality of service without affecting the transmission range, data rate, or contention window. We believe that a cooperative approach that uses vehicles' embedded sensors does not degrade these parameters while increasing the communication range and awareness of the cooperative vehicles while decrease the channel congestion.

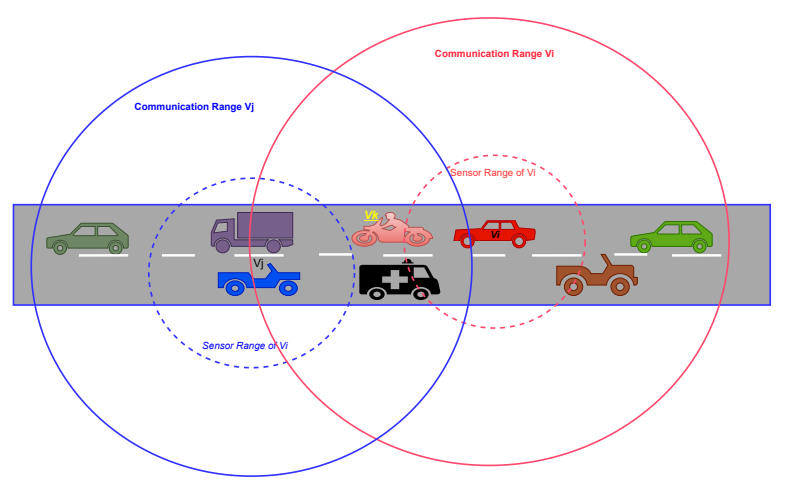

Figure 1. eECAM(-P) working mechanism

\section{ECAM extensions}

In this section we explain the working of our both proposed beaconing schemes, eECAM and eECAM-P. The enhanced Extended CAM (eECAM) adds new dynamic features of reliability and efficiency in the ECAM [1] scheme and provides a more enhanced collective perception approach by sharing the precise information collected from embedded sensors among cooperative vehicles. The eECAM applies a probability based dynamic cooperative priority beaconing approach as its working backbone. The ambition is to take advantage of some safety features using sensors integrated to recent vehicles, such as cameras, radars, acoustic sensors, lidars, etc. to provide a more precise vision of the environment. Basically, a vehicle can send very precise information on the position and speed of other vehicles in its vicinity to increase their knowledge about the environment, thanks to the data provided by these embedded sensors. In this paper, we will illustrate how eECAM can result in a reduction of congestion and contention on the access channel while extending the awareness in the participating vehicles beyond their Field of View (FoV). Also we will show the effectiveness of eECAM-P in expending the view of vehicles in more vast area to leverage more time to the drivers to react in emergency situations.

As shown in Figure 1, the perception of cooperative vehicles is represented by two ranges: a detection range and a communication range. The detection range is the perception view of vehicles collected by the on-board sensors of each vehicle, with a given accuracy, all vehicles within its detection range. This detection range firmly depends on the used technology in the embedded sensors. Where as, the communication range is the perception of cooperative 
vehicles that is collected through received eECAM messages generated by the cooperative vehicles. This communication range depends on several factors, such as density of vehicles, speed, and the frequency of beacons generated by each vehicle. The eECAM implements a probability based

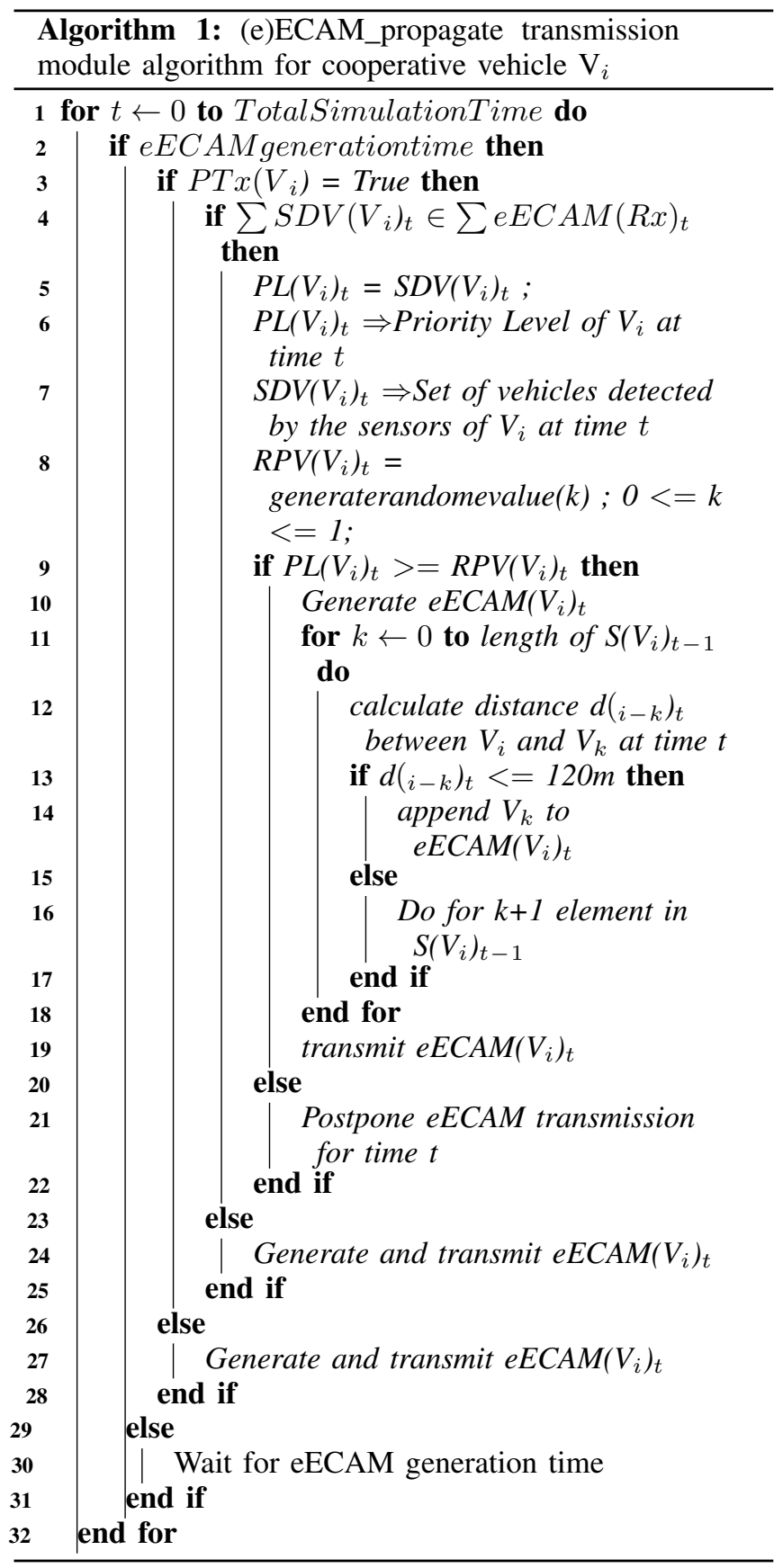

dynamic cooperative priority beaconing scheme alongwith the two folded mechanism of ECAM proposed in [1] to obtain its objective. As shown in the Algorithm- 1 that when a cooperative vehicle (e.g. vehicle $\mathrm{V}_{i}$ ) generates an eECAM message, first it verifies if its eECAM transmission flag $\left(\operatorname{PTx}\left(\mathrm{V}_{i}\right)\right.$ is enabled (Defined by the reception module) so

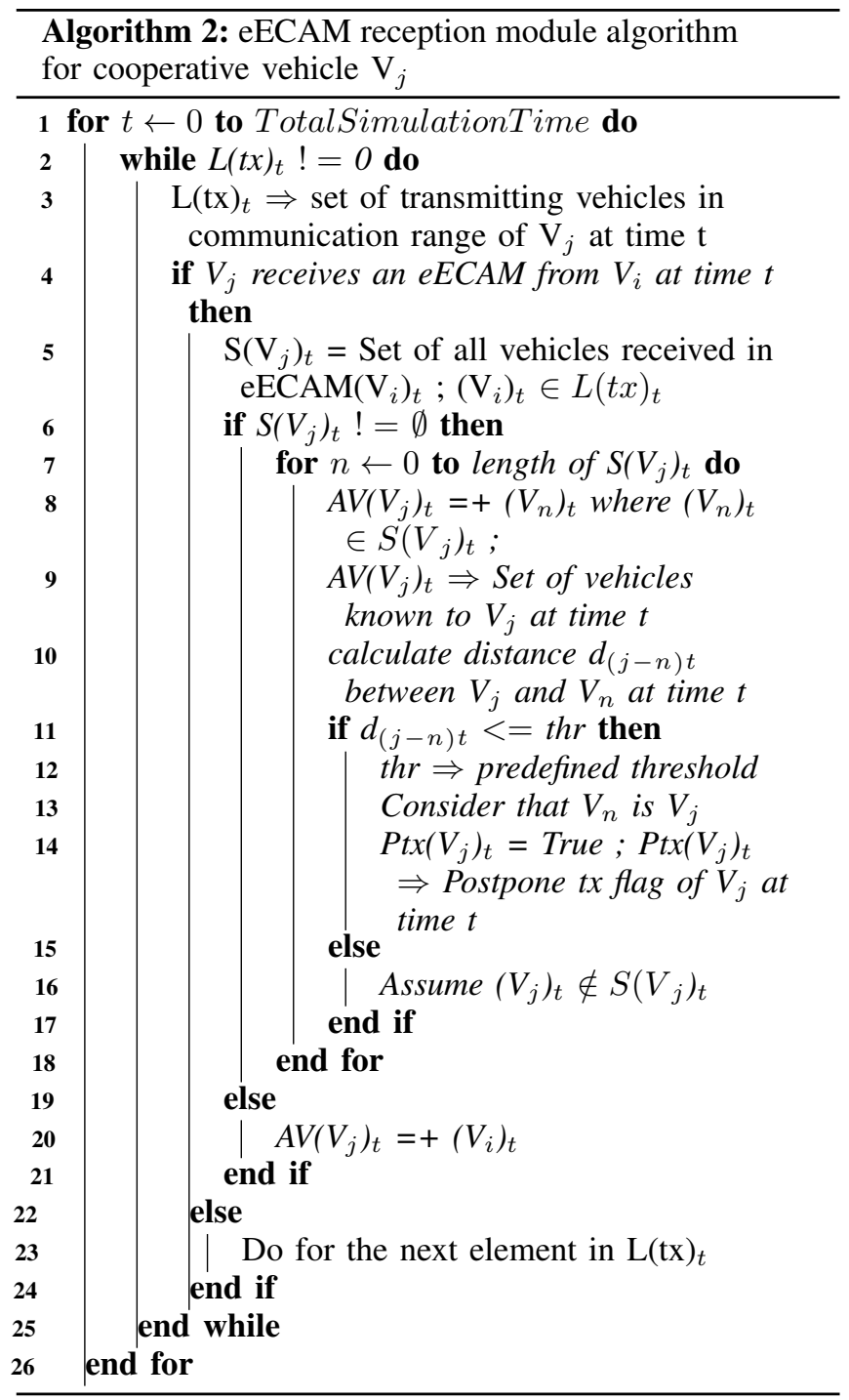

it collects information (position) about vehicles within its sensor detection range and applies the check that whether it has received information about all these neighbor vehicles (Sensor Detected Vehicles (SDV)) in the received messages during the previous transmission time period (in our case that is $100 \mathrm{~ms}$ ) if any of these neighbors is missing so the cooperative vehicle- $\mathrm{V}_{i}$ adds all its detected vehicle's information in its eECAM along with its own.

In the second phase of its working, the cooperative vehicle- $\mathrm{V}_{i}$ applies a probability based dynamic priority scheme to define its transmission priority. As a part of this phase, cooperative vehicle- $\mathrm{V}_{i}$ calculates a transmission priority level $\left(\mathrm{PL}\left(\mathrm{V}_{i}\right)_{t}\right)$ at time $\mathrm{t}$ through the equation- 1 and also generates a random value $\left(\operatorname{RPV}\left(\mathrm{V}_{i}\right)_{t}\right)$ between $0-1$. If the priority level $\left(\operatorname{PL}\left(\mathrm{V}_{i}\right)_{t}\right)$ was greater than $\operatorname{RPV}\left(\mathrm{V}_{i}\right)_{t}$ so the cooperative vehicle- $\mathrm{V}_{i}$ assumes that it has better sensing facilities than other cooperative vehicles in its vicinity and decides to transmit else it postpones the transmission of beacon for the current transmission period. 


$$
P L\left(V_{\mathrm{i}}\right)_{\mathrm{t}}=1-\left(1 /\left(1+\left|S D V\left(V_{\mathrm{i}}\right)_{\mathrm{t}}\right|\right)\right.
$$

On the receiving module of eECAM as shown in algorithm-2, when a cooperative vehicle (e.g. vehicle- $\mathrm{V}_{j}$ ) receives an eECAM packet (for example, from vehicle$\mathrm{V}_{i}$ ), it extricates the list of vehicles included in that packet $\left(\mathrm{S}\left(\mathrm{V}_{j}\right)_{t}\right)$. If the list $\mathrm{S}\left(\mathrm{V}_{j}\right)_{t}$ is empty, then vehicle- $\mathrm{V}_{j}$ enumerates its $\mathrm{FoV}$ list $\mathrm{AV}\left(\mathrm{V}_{j}\right)_{t}$ with the only information of the vehicle- $\mathrm{V}_{i}$ as a neighbor vehicle.

Otherwise, it calculates the distance of each vehicle- $\mathrm{V}_{n}$ in the list $\mathrm{S}\left(\mathrm{V}_{j}\right)_{t}$ from itself. If one of the computed distances is less than a given threshold then it assumes that this entry corresponds to its own position. In this case, vehicle$\mathrm{V}_{j}$ updates its $\mathrm{FoV}\left(\mathrm{AV}\left(\mathrm{V}_{j}\right)_{t}\right)$ by adding all announced vehicles, in addition to vehicle- $\mathrm{V}_{i}$, as neighbor vehicles. Also, it enables its postpone transmission flag $\left(\operatorname{Ptx}\left(\mathrm{V}_{j}\right)_{t}\right)$ to further used in the transmission module eECAM sheme. In-fact, this flag defines that the cooperative vehicles in the communication range are aware of vehicle- $\mathrm{V}_{j}$ at that corresponding time $t$, announced by some other cooperative vehicle (vehicle- $\mathrm{V}_{i}$ ) in the network, thanks to the eECAM.

In addition to the above described probability based dynamic cooperative priority mechanism of eECAM, we have enriched it with an additional robust and more extended view mechanism, named as eECAM with propagation (eECAM-P). Here the cooperative vehicles exert proliferating scheme to propagate the information received in the eECAM beacon. Before a beacon transmission, the cooperative vehicle calculates its maximum communication range, which is the distance between itself and farthest transmitting vehicle from which it has received a beacon message in each transmission time period. Then it propagates the information of cooperative vehicles received through previous eECAM, which are within its communication range, along with its own sensor detected data. This simple but robust proliferating method provides an added feature to increases the awareness of cooperative vehicles beyond their field of view while extensively decreases the load on the channel.

In this way, eECAM(P) reduces the number of beacon transmissions at a sufficient level and increases the awareness of the cooperative vehicles in the network, in result reduces the channel contention leveraging the more vehicles to participate with improved quality of service.

\section{Performance Evaluation}

\subsection{Methodology}

We have verified the effectiveness of our proposed probability based dynamic cooperative priority beaconing scheme (eECAM) and its extended version, eECAM-P, through extensive assessment studies based on a microscopic mobility simulator, Simulation of Urban Mobility (SUMO) version-1.6.0 and discrete-event Network Simulator-3 (NS3 ) version-3.30.1 simulation tools. We have tested this in a Manhattan mobility topology model of $1000 \mathrm{~m}^{2}$ area having 10 x 10 blocks, using SUMO and generated vehicle traffic density of $50,100,200,300,400$, and 500 cooperative vehicles that are concurrently present in the simulation topology through out the simulation time (i.e. 100s). We have generated the flow of vehicles using the ramdonTrips generator, a facility available in SUMO, which apply a uniform distribution function for the position of vehicles. We confirmed that the density of vehicles is constant throughout the simulation time for each scenario. We kept he average speed of the vehicles at $15 \mathrm{~m} / \mathrm{s}$, which is generally maximum speed limit in the urban environment. We converted the vehicle's mobility traces compatible to use in NS-3 using SUMO built-in facility.

\begin{tabular}{ll}
\hline Simulation parameter & Value \\
\hline Simulation Time & $100 \mathrm{~s}$ \\
Simulation Area & $1000 \mathrm{x} 1000 \mathrm{~m}$ \\
Mobility Model & Manhattan Mobility Model \\
Vehicle Average Speed & $50 \mathrm{~km} / \mathrm{h}$ \\
Number of Runs (per scenario) & 20 \\
Packet Size & $200 \mathrm{bytes}$ \\
Perceived Node data size & $20 \mathrm{bytes}$ per node \\
Data rate & $6 \mathrm{Mbps}$ \\
Bandwidth & $10 \mathrm{MHz}$ \\
Transmission power & $7.5 \mathrm{dbm}$ \\
Beacon Interval & $100 \mathrm{~ms}$ \\
Sensor's detection range & $50 \mathrm{~m}$ \\
\hline
\end{tabular}

The network infrastructure among the vehicles was created in the NS-3 simulator. We have used the IEEE-802.11p OFDM physical model with a $6 \mathrm{Mbps}$ data rate and Friis propagation loss model. (e)ECAM messages were generated proportionately to the standard interval of $100 \mathrm{~ms}$. The detection range of Vehicles embedded sensors was set to 50 meters. The simulations were run for total time of 100 seconds for each scenario. For more accurate results, all the scenarios were run 20 times and results were averaged on 20. We ran all these simulations in parallel on a cluster of servers, but it took several days to complete and generated a immense amount of data. A detail of important simulation parameters is shown in table-1. Finally, for the simplicity we use (e)(E)CAM-(P) annotation in the remaining sections to denote CAM and ECAM, eECAM and eECAM-P schemes indifferently.

\subsection{Results Analysis}

We have evaluated the performance of our proposed probability based dynamic priority beaconing scheme in terms of awareness level of the cooperative vehicles, contention on communication channel and average communication range.

The awareness level is the knowledge of the vehicles obtained from the (e)(E)CAM-(P) broadcast messages. Basically this shows the accuracy of the perception of the cooperative vehicles provided by the beaconing mechanism. So this awareness accuracy is computed from an optimal perception reference model which was calculated from the SUMO generated mobility traces. This optimal reference 
model allows us to arbitrate the set of neighboring vehicles present within a given radius, which we have set to 120 meters, of each vehicle at each time period as shown in equation-1.

$$
A_{t}(v)=\frac{\left\|\hat{S}_{t}(v)\right\|}{\left\|S_{t}^{*}(v)\right\|}
$$

Here the $A_{t}(v)$ denotes the awareness of the cooperative vehicle $v$ at each transmission time $t$ where as the set $\hat{S}_{t}(v)$ represents the list of surrounding vehicles collected from the (e)(E)CAM-(P) beaconing scheme and the $S_{t}^{*}(v)$ denotes the optimal awareness set of neighboring vehicles for the cooperative vehicle $v$ at a certain time period $t$. This optimal set of neighboring vehicles $\left(S_{t}^{*}(v)\right)$ comprise of neighboring vehicles present within a given radius (120 meters in our case).

The average awareness level for different vehicle densities is shown in figure-2. The density is the function of all communicating vehicles available in the simulation area throughout the all simulation time. As we can observe from the figure-2 that different variants of our proposed beaconing scheme has shown high performance in terms of awareness level compared with the classical beaconing scheme (CAM) [14]. The ECAM [1] shows $+5.56 \%$ for 50 and $+92.64 \%$ for 500 vehicles of awareness increased and the ECAM-P, ECAM [1] combined with propagating mechanism (propagate with in 120 meters radius), shows an increase from $+23.86 \%$ up to $+104.24 \%$ for the low density (50 vehicles) and high density (500 vehicles) respectively. On the other end eECAM increased the awareness level $+11.51 \%$ for 50 and $+98.98 \%$ for 500 vehicles. Due to its more intransigent transmission mechanism of eECAM the awareness has decreased compare to ECAM-P. In the same way when we enable the information piggybacking mechanism (with in 120meters surrounding) along with eECAM (eECAM-P), the awareness level has increased $+16.14 \%$ for low density and $+107.63 \%$ for the high density. An other variant eECAM-P_dR, which piggybacks the information within its dynamically calculated current maximum communication range at each transmission time period, improved the awareness $+15.27 \%$ and $+100.0 \%$ for low and dense environments accordingly.

The above observations can be substantiated from figure3 , the representation of the degree of nodes, which is the driving force of the awareness level of cooperative vehicles in the network. The degree of nodes for ECAM and ECAM-P is $+5.52 \%$ and $+23.84 \%$ high for low density and $+92.66 \%$ and $+104.2 \%$ high for high density than the classical CAM respectively. The increase in the degree of nodes of eECAM is $+11.4 \%$ and $+98.94 \%$ for low and high densities respectively however if the piggybacking is enabled with eECAM (eECAM-P) than the improvement of the degree of nodes reaches from $+16.18 \%$ to $+107.6 \%$ for 50 and 500 vehicles accordingly. In the same way eECAMP_dR enhanced the degree $+15.26 \%$ for 50 vehicles and $+100.02 \%$ for the 500 vehicles environment.

Furthermore, the figure- 4 and figure- 5 give a detailed view of the channel contention, observed by these beacon-

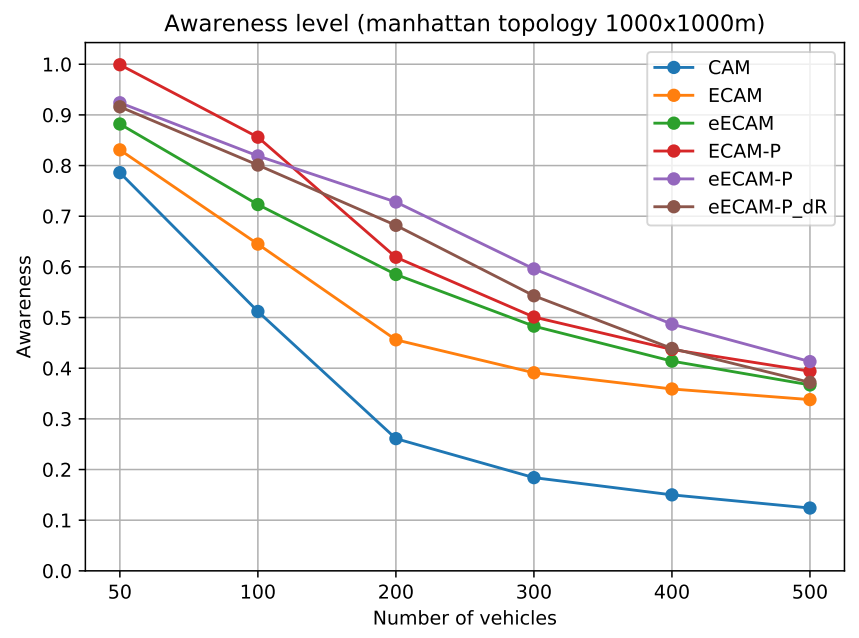

Figure 2. Awareness level

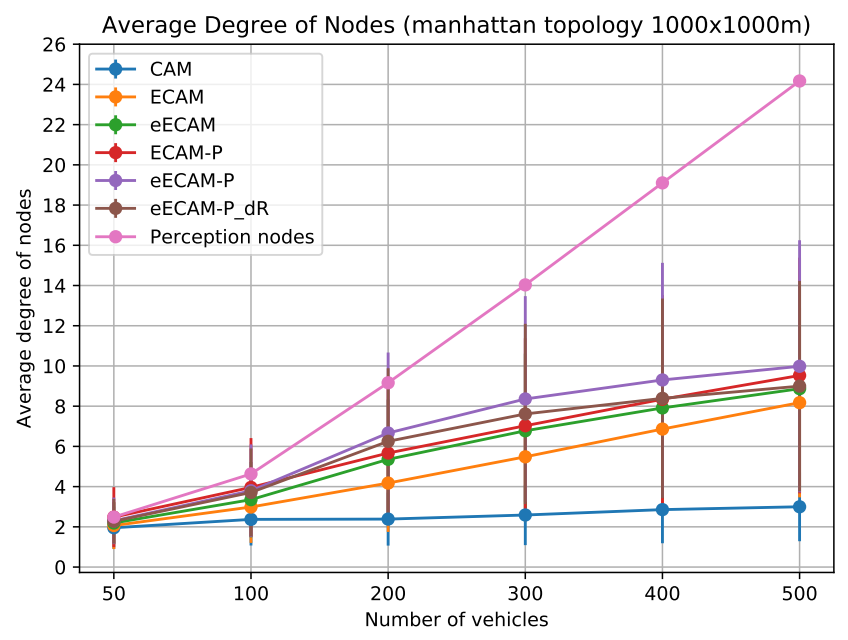

Figure 3. Degree of nodes

ing schemes. We have calculated the channel contention by counting the total number of transmissions/receptions through out the simulation time and averaged them on 20. The figure- 4 indicates that in the high dense scenarios, the number of transmissions is very high for classical CAM where as the ECAM reduces the number of transmissions $19.66 \%$ for the low density and up to $96.7 \%$ in the high dense scenarios. Also the ECAM-P transmitted $42.98 \%$ less beacons for 50 and $100.5 \%$ less for 500 cooperative vehicles. In the same way, eECAM reduces the contention immensely by curtailing the transmissions by $153.7 \%$ and $96.3 \%$ for low and high dense cooperative scenarios respectively. In the eECAM-P variant this number decreases upto $155.3 \%$ for 50 cooperative vehicles and $100.47 \%$ for the density of 500 cooperative vehicles where as the eECAMP_dR dwindles this percentage to $154.3 \%$ for low dense areas and $-96.18 \%$ for the highly dense circumstances. 
This reduction of channel load gives opportunity to more cooperative vehicles to participate in the communication, thanks to our proposed probability based dynamic priority beaconing scheme and its variants.

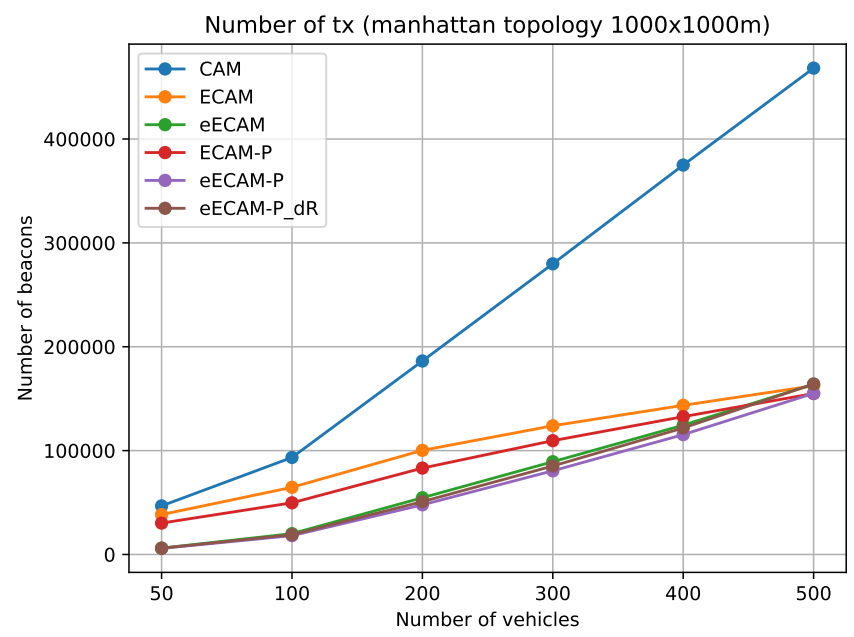

Figure 4. Number of transmitted beacons

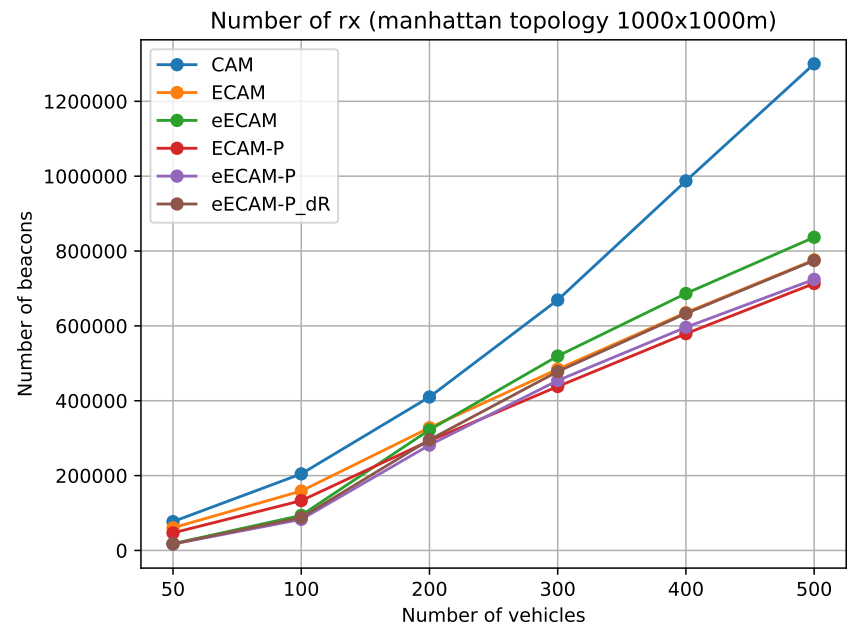

Figure 5. Number of received beacons

We have also examined the performance of (e)(E)CAM(P) in terms of communication range for different density networks. The communication range is described as the distance between transmitter and its receiver for each beacon message. Average and maximum communication ranges are shown in figure-6. The maximum communication range is the highest distance observed from a transmitter to the receiver in a simulation run.

\section{Conclusion}

In this paper, we have presented a probability based dynamic priority beaconing mechanism (eECAM-P) to increase the perception of cooperative vehicles utilizing the

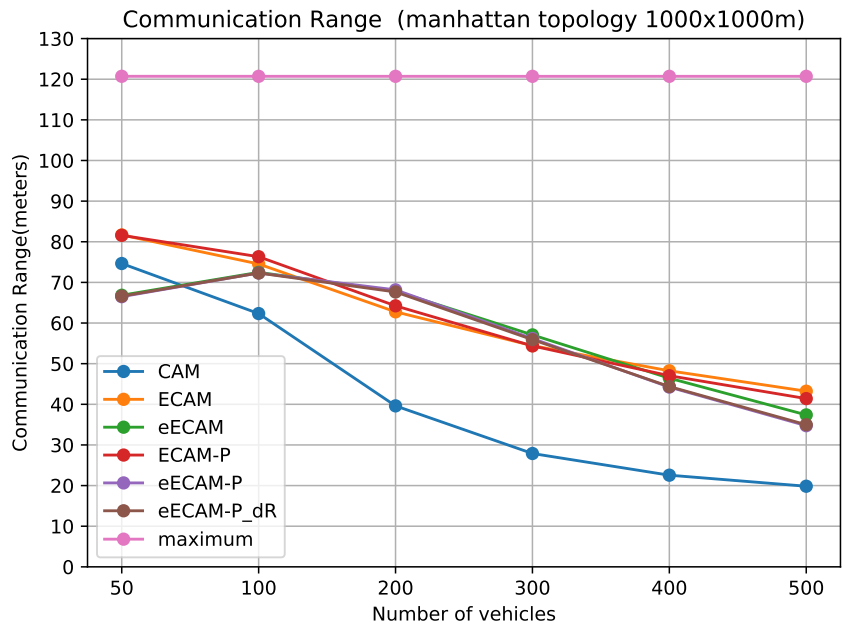

Figure 6. Communication range

vehicle's on-board sensors. Our proposed eECAM-P scheme manipulates the sensor collected data of the neighboring vehicles to increase the perception of cooperative vehicles beyond their field of view and decreases the beacons transmission. We have analysed the performance of the different versions of eECAM-P for various vehicle density environments. These simulation results proved the effectiveness of our proposed scheme in enhancing the perception of cooperative vehicles while successfully reducing the contention on the communication channel upto an optimal level. This more accurate and increased perception leverages the cooperative vehicles with more reaction time in case of any unexpected event on the other hand the curtailed channel congestion gives opportunity to more vehicles to participate in the communication network, thanks to the vehicle's embedded sensors.

\section{References}

[1] Sadia Ingrachen, Nadjib Achir, Paul Muhlethaler, Tounsia Djamah, Amine Berqia. A Collaborative Environment Perception Approach for Vehicular Ad hoc Networks. VTC 2018 - Fall - 2018 IEEE 88th Vehicular Technology Conference, Aug 2018, Chicago, United States.

[2] "Standard for Information Technology - Telecommunications and Information Exchange between Systems - Local and Metropolitan Area Networks - Specific Requirements Part 11 - Amendment 6: Wireless Access in Vehicular Environment," IEEE, 2010

[3] "Intelligent Transport Systems (ITS); Access layer specification for Intelligent Transport Systems operating in the $5 \mathrm{GHz}$ frequency band" ETSI EN 302663 V1.2.0 (2012-11)

[4] Kan Zheng, Qiang Zheng, Haojun Yang, Long Zhao, Lu Hou, and Periklis Chatzimisios. "Reliable and Efficient Autonomous Driving: The Need for Heterogeneous Vehicular Networks" IEEE Communications Magazine, p.72-79 (Volume: 53, Issue:12, Dec-2015)

[5] Chetan Belagal Math, Ahmet Ozgur, Sonia Heemstra de Groot, Hong $\mathrm{Li}$, "Data Rate Based Congestion Control in V2V Communication for Traffic Safety Applications" (IEEE-SCVT) November-2015.

[6] Hendrik-Jörn Günther, Björn Mennenga, Oliver Trauer, Raphael Riebl, and Lars Wolf. Realizing collective perception in a vehicle. In Vehicular Networking Conference (VNC), 2016 IEEE, pages 1-8. IEEE, 2016. 
[7] Hendrik-Jorn Günther, Raphael Riebl, Lars Wolf, Christian Facchi, "Collective perception and decentralized congestion control in vehicular ad-hoc networks". IEEE Vehicular Networking Conference(VNCDecember-2016).

[8] Fuxin Zhang, Yuyue Du, Wei Liu, Peng Li, "Model Predictive Power Control for Cooperative Vehicle Safety Systems". IEEE Access, pages.4797 - 4810, January, 2019.

[9] A. Veksler, T. A. Johansen, F. Borrelli, B. Realfsen. "Dynamic Positioning With Model Predictive Control," IEEE T CONTR SYST T , vol. 24, no. 4, pp. 1340-1353, 2016.

[10] Kloiber, B.; Harri, J.; Strang, T.; Sand, S.; Garcia, C.R. Random transmit power control for DSRC and its application to cooperative safety. IEEE Trans. Dependable Secur. Comput. 2016, 13, 18-31.

[11] Torrent-Moreno M, Santi P, Hartenstein H . "Distributed fair transmit power adjustment for vehicular ad hoc networks. In: Sensor and ad hoc communications and networks". 2006-SECON'06. 3rd annual IEEE communications society on, Reston, VA; 2006. p. 479-88 .

[12] M. A. Javed and J. Y. Khan, "A Cooperative Safety Zone Approach to Enhance the Performance of VANET Applications," 2013 IEEE 77th Vehicular Technology Conference (VTC Spring), Dresden, 2013, pp. 1-5.

[13] A. Patil, M. Deeksha, N. Shekar, V. Shet and M. Kulkarni, "Transmit Data Rate Control Based Decentralized Congestion Control Mechanism for VANETs," 2019 International Conference on Data Science and Communication (IconDSC), Bangalore, India, 2019, pp. 1-5, doi: 10.1109/IconDSC.2019.8816902.

[14] ETSI EN 302 637-2 V1.3.2 (2014-11), Intelligent Transport Systems (ITS); Vehicular Communications, Basic Set of Applications; Part 2: Specification of Cooperative Awareness Basic Service. 